\title{
INVERSIVE AND CONFORMAL CONVEXITY
}

\section{P. J. KELLY AND E. G. STRAUS ${ }^{1}$}

1. Introduction. A set $S$ in Euclidean $E_{n}$-or, equivalently, on the $n$-sphere $S_{n}$-is called inversively convex if there is an inversion on an $(n-1)$-sphere in the space which transforms $S$ into a convex set. A simple closed $(n-1)$-surface is inversively convex if one of the two regions bounded by it is inversively convex.

The group generated by the inversions can be characterized as follows $[1$, p. 102].

LEMMA 1. Let $T$ be a product of inversions of $E_{n}$. Then $T$ is either a similarity or the product of an isometry and an inversion. The product of two concentric inversions is a similarity.

Proof. If $T$ keeps the point at infinity fixed then it transforms the planes (spheres through infinity) into planes and is therefore affine. Since $T$ is also conformal it is therefore a similarity.

Now let $T$ transform infinity into the finite point $p$ and let $I_{p}$ be an inversion on a sphere with center $p$ (inversion at $p$ for short). Then $I_{p} T$ keeps infinity fixed and is a similarity; or, by a suitable choice of radius for the sphere of $I_{p}$, an isometry $U$. Thus $T=I_{p} U$. The last statement of the lemma follows from the fact that such a product leaves infinity fixed.

As a consequence of Lemma 1 we see that inversive convexity is equivalent to convexity under an element of the group $G$ generated by inversions. In the (complex) plane (Riemann sphere) $G$ is the group of conformal and anticonformal linear fractional transformations. For $n>2$ the group $G$ is the group of all conformal transformations $[1$, p. 100] and inversive convexity is therefore equivalent to conformal convexity. As another consequence of Lemma 1 we may now speak of inversive convexity at $p$, meaning convexity under inversion on the spheres with center $p$.

In $\S 2$ we give characterizations of inversive convexity of sets and curves in $E_{2}\left(S_{2}\right)$. Moreover, we characterize the centers of inversion which transform a given set into a convex set. In $\$ 3$ new characterizations are given which are valid also for $n>2$ to yield a characterization of conformal convexity in $E_{n}\left(S_{n}\right)$.

2. Inversive convexity in the plane. A first simple characterization

Received by the editors July 5, 1956.

1 The first author was supported in part by the Ford Foundation. The second author was supported in part by the National Science Foundation. 
of inversive convexity at $p$, valid regardless of dimension, is given by the following.

THEOREM 1. The set $S$ is inversively convex at $p$ if and only if for every two points $a, b \in S$ the arc ab of the circle determined by $a, b, p$ which does not contain $p$, is contained in $S$. (If $p=a$ this reduces to the statement that $p, b$ are joined by some circular arc in $S$.)

The proof is obvious if we remember that the arc $a b$ in question goes into the segment $a^{\prime} b^{\prime}$ of $S^{\prime}$, where $x^{\prime}$ indicates the image of $x$ upon inversion at $p$.

From Theorem 1 we see that inversive convexity implies a certain kind of circle convexity. If $S$ is inversively convex at two points $p, q$ then we get a "lune convexity" from Theorem 1 .

In order to obtain a more useful characterization we now pass to the question of inversive convexity of curves. A curve $C$ in $E_{2}\left(S_{2}\right)$ is called convex if it lies in the boundary of its convex hull. A convex curve is thus either a simple arc (possibly with one endpoint at infinity) or a simple closed curve (possibly through infinity).

Lemma 2. An oriented simple curve $C$ in the plane is convex if and only if all triangles $\triangle a b c$ with vertices appearing in that order on $C$ have the same orientation. (Collinear triples may be excluded or permitted according as we wish to define strict convexity or not.)

Proof. The interior of $\triangle a b c$ lies in the convex hull $H(C)$ of $C$. Thus the orientation given to the boundary of $H(C)$ by that of $C$ is the same as that of $\triangle a b c$.

Conversely if $C$ is not convex then the boundary of $H(C)$ contains at least three points $a, b, c$ of $C$ so that $\triangle a b c$ contains in its interior a point $d \in C$. The four triangles determined by $a, b, c, d$ can then not have the same orientation.

Now, on the simple, oriented curve $C$, each triple of points $a, b, c$ determines a circle $K_{a b c}$, whose orientation is given by the order given to $a, b, c$ by the orientation of $C$. We label the open (closed) side of $K_{a b c}$ corresponding to one orientation of the plane by $S_{o}^{+}(a, b, c)$ (respectively $S_{c}^{+}(a, b, c)$ ) and the open (closed) side corresponding to the opposite orientation by $S_{o}^{-}(a, b, c)$ (respectively $S_{c}^{-}(a, b, c)$ ). We now define the intersections

$$
\begin{array}{ll}
S_{o}^{+}=\bigcap_{a, b, c \in C} S_{o}^{+}(a, b, c) ; & S_{c}^{+}=\bigcap_{a, b, c \in C} S_{c}^{+}(a, b, c), \\
S_{o}^{-}=\bigcap_{a, b, c \in C} S_{o}^{-}(a, b, c) ; & S_{c}^{-}=\bigcap_{a, b, c \in C} S_{c}^{-}(a, b, c) .
\end{array}
$$


Since inversion at a point $p$ preserves the orientation of every circle containing $p$ in its interior and reverses the orientation of every circle containing $p$ in its exterior, Lemma 2 yields the following.

TheOREM 2. A simple, oriented curve $C$ becomes convex upon inversion at $p$ if and only if $p \in S_{c}^{+} \cup S_{c}^{-}$, and becomes strictly convex if and only if $p \in S_{o}^{+} \cup S_{o}^{-}$.

Proof. From our construction we see that a point $p$ lies in $S_{c}^{+}$or $S_{c}^{-}$if and only if it lies in the closed interior of all $K_{a b c}$ with one orientation of $\triangle a b c$ and in the closed exterior of all $K_{a b c}$ with the opposite orientation of $\triangle a b c$. Thus by the remark preceding the theorem, upon inversion at $p$ the image triples which are not collinear will be triangles which all have the same orientation. Thus according to Lemma 2 the image $C^{\prime}$ is convex. If $p \in S_{o}^{+} \cup S_{o}^{-}$then no triple $a, b, c$ lies on a circle through $p$. Thus collinearity of $a^{\prime}, b^{\prime}, c^{\prime}$ is excluded and $C^{\prime}$ is strictly convex.

Conversely if $p$ lies on opposite sides of $K_{a b c}$ and $K_{\text {def }}$ then the images $\triangle a^{\prime} b^{\prime} c^{\prime}$ and $\triangle d^{\prime} e^{\prime} f^{\prime}$ upon inversion at $p$ have opposite orientation and $C^{\prime}$ is not convex. Finally if $p \in\left(S_{c}^{+}-S_{o}^{+}\right) \cup\left(S_{c}^{-}-S_{o}^{-}\right)$then $p$ lies on one of the circles $K_{a b c}$. Thus the image $C^{\prime}$ contains collinear $a^{\prime}, b^{\prime}, c^{\prime}$ and is not strictly convex.

Corollary. Let $S$ be the exterior of a closed convex curve $C$ (possibly through infinity) and $P$ the set of points at which $S$ is inversively convex, then $P$ is a convex (possibly empty) closed subset of the closed interior of $C$.

Instead of convexity we now consider the concept of local convexity of a curve.

Definition. An oriented curve $C$ is locally convex if every point $x$ on $C$ is contained in an open convex arc $C_{x}$ of $C$ so that the convex hulls $H\left(C_{x}\right)$ lie on the same side of $C$. A curve $C$ is inversively locally convex if its image upon inversion on some circle is locally convex.

If the $C_{x}$ are strictly convex then the condition on $H\left(C_{x}\right)$ becomes superfluous.

A locally convex curve need not be convex and need not even be simple. However a simple closed curve (possibly through infinity) which is locally convex is convex. Thus for simple closed curves the characterization to be given of inversive local convexity is a characterization of inversive convexity.

Definition. A limit circle of a curve $C$ at a point $x \in C$ is the limit of a sequence of circles $K_{a b c}$ with $a, b, c \in C$ as the arc $a, b, c$ shrinks to the point $x$. If $C$ is oriented then the approximating circles are oriented and we obtain oriented limit circles (possibly of zero radius). 
Lemma 3. An oriented curve $C$ is locally convex if and only if all its limit circles have the same orientation.

Proof. Each point $x \in C$ is contained in a convex subarc $C_{x} \subset C$. Hence by Lemma 2 all $K_{a b c}$ with $a, b, c \in C_{x}$ have the same orientation, which is also the orientation of any limit circle at $x$. Now let $x, y$ be two points on $C$. By hypothesis $C_{x}$ and $C_{y}$ have the same orientation relative to their convex hulls. Hence the limit circles at $x$ and $y$ have the same orientation.

Assume now that $C$ is not locally convex. Then either (i) there exists a point $x \in C$ so that every open subarc $C_{x} \subset C$ which contains $x$, contains points $a, b, c$ so that $x$ is an interior point of $\triangle a b c$; or (ii) $C$ contains a straight line segment $x y$ so that $H\left(C_{x}\right), H\left(C_{y}\right)$ are on opposite sides of $x y$. In case (i) not all the circles determined by three of the four points $a, b, c, x$ can have the same orientation. If we let $C_{x}$ shrink to $x$ we thus obtain limit circles at $x$ with opposite orientations. In case (ii) the limit circles of $C_{x}$ and of $C_{y}$ are on opposite sides of $C$.

Theorem 3. Let $C$ be an oriented curve. Let $S_{c}^{+}$be the set of points which lie on one closed side of all the limit circles of $C$ and $S_{c}^{-}$the set of points lying on the other closed side of all the limit circles of $C$. Then $C$ is locally convex upon inversion at $p$ if and only if $p \in \mathcal{S}_{c}^{+} \cup \mathcal{S}_{c}^{-}$.

The derivation of Theorem 3 from Lemma 3 is entirely analogous to that of Theorem 2 from Lemma 2 and is therefore omitted.

If $C$ is differentiable the limit circles are the circles of curvature of $C$, and Theorem 3 could then be stated in terms of circles of curvature. Another interesting special case is the following.

CoRollary. If $C$ is a closed convex curve (possibly through infinity), then the intersection $S$ of the closed interiors of the circles $K_{a b c}(a, b, c \in C)$ is also the intersection of the closed interiors of the limit circles of $C$. (The "interior" of a circle of infinite radius is that half plane which contains $C$.) The set $S$ is the set of points at which the exterior of $C$ is inversively convex.

If the curvature of $C$ is bounded away from zero-or, more generally, if the limit circles of $C$ have bounded radius, then the interior of $C$ is inversively convex at every point sufficiently far from $C$.

3. Conformal convexity. Instead of extending the method of $\$ 2$ to higher dimensions-which is possible in part but cumbersome-we introduce a new method which gives new insight also in the twodimensional case.

Definition. A sphere of support of a set $S$ at a point $x$ in the closure 
$\bar{S}$ of $S$ is a sphere $K$ such that $S$ lies entirely on one closed side of $K$. An extremal sphere of support of $S$ at $x$ is a sphere of support of $S$ at $x$ such that the side containing $S$ (we shall call it the positize side) is minimal.

An extremal sphere of support may be a plane or degenerate to a single point. There may be several extremal spheres of support at a point.

Now we associate to each boundary point $x$ of $S$ the set $K_{x}$ consisting of the union of the closed negative sides of the extremal spheres of support of $S$ at $x$. Finally we let $K_{S}$ be the intersection of the $K_{x}$ as $x$ runs through all boundary points of $S$.

Lemma 4. A set $S$ with interior points is inversively convex at $p$ if and only if there is a supporting sphere through $p$ at every boundary point of $S$.

Proof. The image $S^{\prime}$ of $S$ upon inversion at $p$ is convex if and only if there is a plane of support to $S^{\prime}$ at every boundary point of $S^{\prime}$. Inverting again we transform the planes into spheres of support of $S$ through $p$.

TheOREM 4. A set $S$ with interior points is inversively convex at $p$ if and only if $p \in K_{s}$. Thus inversive convexity of $S$ is equivalent to the nonemptiness of $K_{S}$.

Proof. If $S$ is inversively convex at $p$ then according to Lemma 4 there is a sphere of support to $S$ through $p$ at every boundary point $x$ of $S$. This sphere is contained in the closed negative side of some extremal sphere of support to $S$ at $x$ and hence $p \in K_{x}$ for every boundary point $x$, that is $p \in K_{S}$.

Conversely, assume $p \in K_{S}$, then for every boundary point $x$ of $S$ there is an extremal sphere of support $K$ which contains $p$ in its closed negative side. The sphere through $p$ and tangent to $K$ at $x$ is therefore in the closed negative side of $K$ and is a sphere of support to $S$ at $x$ (in case $x=p$ this choice is not unique). Thus, according to Lemma $4, S$ is inversively convex at $p$.

A combination of Theorem 4 with the Corollary to Theorem 3 yields some information about convex curves, (the analogous theorem also holds in higher dimensions).

Corollary. For a closed convex twice differentiable curve $C$ in the plane the following sets are identical:

(i) The intersection of the closed interiors of the circles of curvature of $C$, and the intersection of the closed interiors of the maximal inscribed circles to $C$. 
(ii) The intersection of the closed exteriors of the circles of curvature of $C$, and the intersection of the closed exteriors of the minimal circumscribed circles to $C$.

\title{
REFERENCE
}

1. W. Blaschke, Vorlesungen über Differentialgeometrie I, Berlin, 1930.

University of California at Santa Barbara and Los Angeles and INSTITUTE FOR ADVANCED STUdY

\section{HOMOTOPY GROUPS OF ONE-DIMENSIONAL SPACES}

\author{
M. L. CURTIS ${ }^{1}$ AND M. K. FORT, JR.
}

In this paper we prove the following theorem:

If $S$ is a one-dimensional separable metric space, then $\pi_{k}(S)=0$ for all $k>1$.

Actually it is proved that a much broader class of spaces than spheres have the property that mappings of these spaces into onedimensional spaces are homotopic to constant maps. This class of spaces includes, for example, projective spaces and Lens spaces.

Lemma $1 .^{2}$ Let $X$ be a compact metric space whose one-dimensional integral singular homology group is a torsion group. Then for any finite covering $G$ of order one by arcwise-connected open sets, $G$ does not contain a simple loop.

Proof. By a simple loop we mean a simple chain such that the first and last sets are the same. Let $K$ be the nerve of $G$. Since $K$ is onedimensional, a simple loop in $G$ implies a nonbounding one cycle in $K$. Hence it suffices to show that $H_{1}(K)=0$.

Let $\phi: X \rightarrow K$ be a canonical map. For each vertex $v$ in $K$ we choose a point $\psi(v)$ in the element of $G$ corresponding to $v$. For each edge $\sigma$ with vertices $v_{1}$ and $v_{2}$ we extend $\psi$ on $\left\{v_{1}, v_{2}\right\}$ to a mapping of $\sigma$ into the union of the two elements of $G$ corresponding to $v_{1}$ and $v_{2}$. This is possible, since these two elements of $G$ are arcwise connected and

Received by the editors August 22, 1956 and, in revised form, September 28, 1956.

1 Supported by contract AF 18(600)-1571.

2 The method of proof used below, which extended this lemma from manifolds $M$ with $H_{1}(M)=0$ to compact spaces $X$ with $H_{1}(X)$ a torsion group, was suggested by the referee. 